\title{
Spectral diffusion time scales in InGaN/GaN quantum dots
}

Cite as: Appl. Phys. Lett. 114, 112109 (2019); https://doi.org/10.1063/1.5088205

Submitted: 09 January 2019 . Accepted: 05 March 2019 . Published Online: 22 March 2019

Kang Gao (D), Helen Springbett (D), Tongtong Zhu (D), Rachel A. Oliver (D), Yasuhiko Arakawa, and Mark J.

Holmes (iD)

\section{ARTICLES YOU MAY BE INTERESTED IN}

Ultrafast bulk carrier dynamics in various GaN crystals at near-infrared wavelengths under one- and two-photon absorption

Applied Physics Letters 114, 112108 (2019); https://doi.org/10.1063/1.5089108

Density control of GaN quantum dots on AIN single crystal

Applied Physics Letters 114, 082101 (2019); https://doi.org/10.1063/1.5083018

Effect of low hole mobility on the efficiency droop of AlGaN nanowire deep ultraviolet light emitting diodes

Applied Physics Letters 114, 101104 (2019); https://doi.org/10.1063/1.5091517

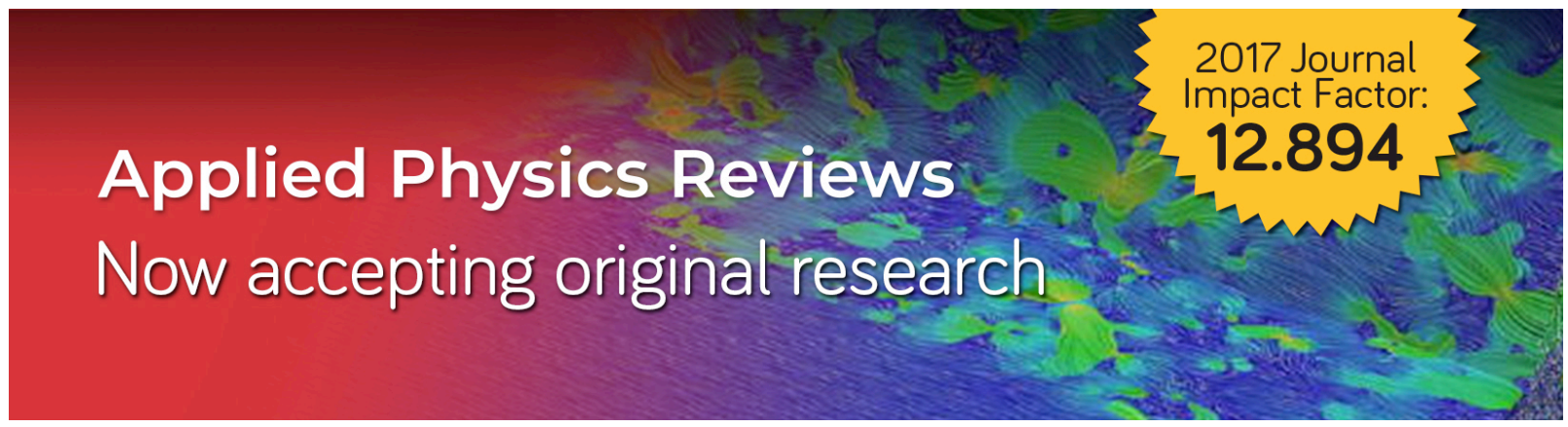




\title{
Spectral diffusion time scales in InGaN/GaN quantum dots
}

\author{
Cite as: Appl. Phys. Lett. 114, 112109 (2019); doi: 10.1063/1.5088205 \\ Submitted: 9 January 2019 . Accepted: 5 March 2019. \\ Published Online: 22 March 2019
}

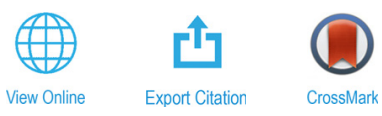

Kang Gao, (D) Helen Springbett, ${ }^{2}$ (D) Tongtong Zhu, ${ }^{2}$ (D) Rachel A. Oliver, ${ }^{2}$ (D) Yasuhiko Arakawa, and Mark J. Holmes ${ }^{1,3, a)}$ (D)

\author{
AFFILIATIONS \\ ${ }^{1}$ Institute for Nano Quantum Information Electronics, The University of Tokyo, 4-6-1 Komaba, Meguro-ku, Tokyo 153-8505, Japan \\ ${ }^{2}$ Department of Materials Science and Metallurgy, The University of Cambridge, 27 Charles Babbage Road, Cambridge CB3 OFS, \\ United Kingdom \\ ${ }^{3}$ Institute of Industrial Science, The University of Tokyo, 4-6-1 Komaba, Meguro-ku, Tokyo 153-8505, Japan
}

a)holmes@iis.u-tokyo.ac.jp

\begin{abstract}
A detailed temporal analysis of the spectral diffusion phenomenon in single photon emitting InGaN/GaN quantum dots (QDs) is performed via measurements of both time-varying emission spectra and single photon emission intensity autocorrelation times. Excitation dependent phenomena are investigated via the optical excitation of carriers into the GaN barrier material and also directly into InGaN. Excitation into InGaN reveals that the fastest environmental fluctuations occur on timescales as long as a few hundreds of nanoseconds: an order of magnitude longer than previously measured in GaN QDs. Such long time scales may in future allow for the generation of indistinguishable photons in spite of the fact that the experimentally measured linewidths are broad.
\end{abstract}

Published under license by AIP Publishing. https://doi.org/10.1063/1.5088205

Quantum dots (QDs) are promising structures for the development of single photon emitting devices ${ }^{1-4}$ and therefore are expected to find applications in several future quantum information processing applications such as teleportation, ${ }^{5}$ computation, ${ }^{6}$ sensing, and quantum key distribution. ${ }^{8}$ As a subset of thus-far investigated QD-based single photon emitters, III-nitride structures are of interest due to their widely tunable emission wavelength range, ${ }^{9-12}$ the possibility of operation at comparatively high temperatures, ${ }^{13-20}$ the possibility of high purity single photon emission, ${ }^{21}$ and also the large scale industrial infrastructure available for manufacturing and processing such materials. However, III-nitride QDs are currently known to suffer from severe spectral diffusion caused by strong Coulomb interactions between QD-confined charges and itinerant charges in the surrounding environment. ${ }^{22-27}$ This spectral diffusion will severely limit (and possibly negate) the possibility of indistinguishable photon generation $^{28-31}$ from these structures, and thus demands a detailed investigation, particularly with regard to the time scales on which it occurs.

In this work, we report excitation dependent measurements of the long-term (occurring on time scales of seconds) and fast temporal scale ( nanosecond) spectral diffusion phenomena in an isolated self-assembled InGaN/GaN QD. The QDs were grown on c-plane GaN/sapphire pseudo-substrates using a modified droplet epitaxy method by $^{32-34}$ betal Organic Chemical Vapour Deposition on a $6 \times 2$ in. Thomas Swan close-coupled showerhead reactor and are situated in an etched micro-pillar structure containing porous DBRs (formed by electrochemical etching) for enhanced emission extraction efficiency. ${ }^{35,36}$ Due to the modified droplet epitaxy growth of the QDs, they form as part of a fragmented quantum well. ${ }^{32,37}$ Characterization of the QDs is performed optically using microphotoluminescence spectroscopy at a temperature of $7 \mathrm{~K}$ in a closed cycle cryostat under continuous wave $(\mathrm{CW})$ laser excitation. Low temperature operation is required to suppress environmental fluctuations as much as possible. We excite the sample through a $50 \times$ objective lens $(\mathrm{NA}=0.42)$ to a spot size of $\sim 2 \mu \mathrm{m}$, and the resulting photoluminescence is then collected with the same objective and collimated before being directed for measurement in a $30 \mathrm{~cm}$ spectrometer with a $1200 \mathrm{~mm}^{-1}$ diffraction grating. The spectrometer has two exit ports: One equipped with a nitrogen cooled CCD for the standard spectral measurement, and the other connected to a Hanbury Brown and Twiss (HBT) type autocorrelation setup, consisting of a beam splitter and two photomultiplier tube (PMT) detectors, for measuring temporal variations of the emission via the second order intensity autocorrelation function 

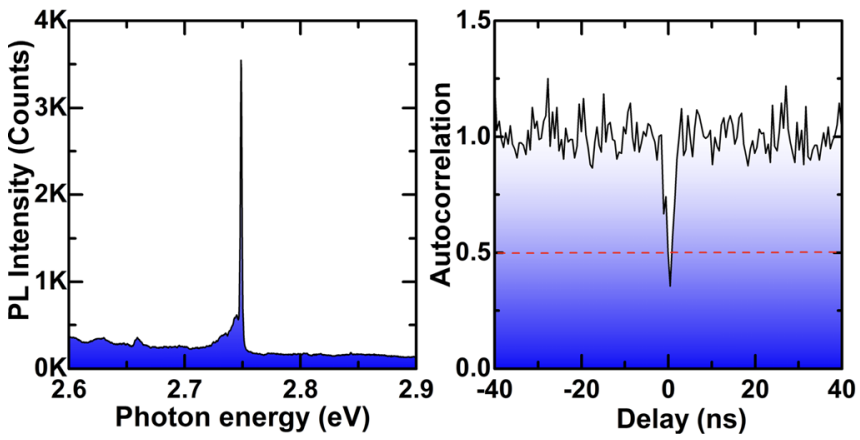

FIG. 1. Microphotoluminescence spectrum and autocorrelation measurement of an isolated InGaN/GaN QD. The measurement of $\mathrm{g}^{(2)}(0)<0.5$ confirms the single photon nature of the emission. For these measurements, the emitter was excited with a $400 \mathrm{~nm}$ laser at a power of $255 \mathrm{~W} \mathrm{~cm}^{-2}$, and emission from the whole peak (excluding the low energy acoustic phonon sideband) was measured for autocorrelation.

$$
g^{(2)}(\tau)=\frac{\langle I(t+\tau) \cdot I(t)\rangle}{\langle I(t)\rangle^{2}} .
$$

The exit slit of the spectrometer for this second port is used for spectral selection (i.e.: the spectrometer is used as a monochromator for filtering with a tunable bandwidth).

We begin by investigating the spectrum of a selected emitter at $\sim 2.74 \mathrm{eV}(450 \mathrm{~nm})$ from which we confirm single photon emission (see Fig. 1) via a $\mathrm{g}^{(2)}(0)$ measurement of 0.36 (below the 2-photon threshold of 0.5). In order to study the spectral stability of this emitter, we first measure and analyze temporal variations in the emission spectrum over a period of $1 \mathrm{~h}$ under excitation at $355 \mathrm{~nm}, 375 \mathrm{~nm}$, and $400 \mathrm{~nm}$ (all experiments are performed at the same excitation power of $\sim 193 \mathrm{~W} \mathrm{~cm}^{-2}$ ). These excitation wavelengths are chosen as they enable us to excite carriers into the surrounding $\mathrm{GaN}$ material $(355 \mathrm{~nm})$ and also directly into the InGaN layer $(375 \mathrm{~nm}$ and $400 \mathrm{~nm})$. In Fig. 2(a), we plot 2D color maps of the emission spectra as they vary with time. In all cases, we observe a clear fluctuation in the emission energy of the dot, occurring on time scales on the order of a few

(a)
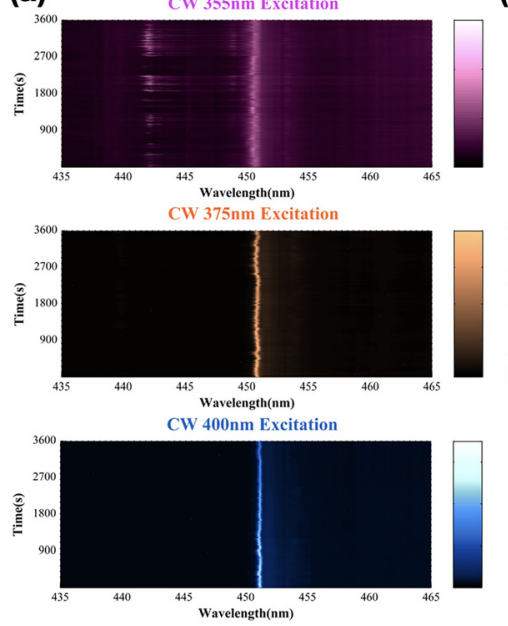

(b)

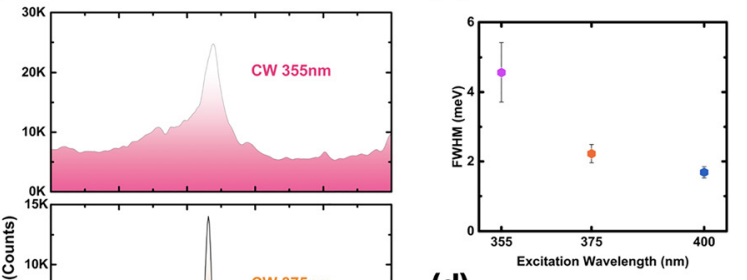

(d)

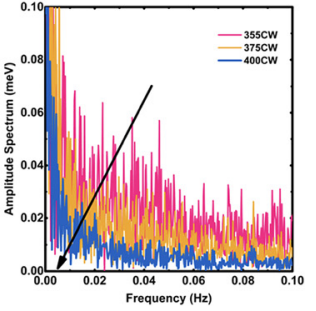

FIG. 2. Microphotoluminescence from the studied single $\ln G a N / G a N$ QD. (a) Temporal variation of the emission spectra of a single emitter measured over a period of $1 \mathrm{~h}$ at excitation wavelengths of $355 \mathrm{~nm}$, $375 \mathrm{~nm}$, and $400 \mathrm{~nm}$ (all the same excitation power of $193 \mathrm{~W} \mathrm{~cm}^{-2}$ ). (b) Integrated emission spectra from the data in (a). (c) Excitation wavelength dependence of the emission linewidth [extracted from the data in (b)]. (d) Fourier transforms of the fluctuations in the peak energies, showing a suppression of the magnitude of the spectral diffusion at all time scales for excitation below the GaN bandgap. 
give access to such fast time scales because the experiment is limited by the data acquisition times of the CCD to time scales greater than several milliseconds. The most important time scales of spectral diffusion in InGaN QDs have therefore not been measured to date. In the following, we investigate the spectral diffusion effects on nano-second time scales for this dot using autocorrelation measurements typically used for single photon characterization. More specifically, we perform photon autocorrelation measurements whilst spectrally selecting only a small part of the emission peak, ${ }^{41}$ as illustrated in Fig. 3. The temporal resolution of this experiment is determined by the combined response functions of our PMTs to be $\sim 370$ ps. By selectively measuring a narrower region of the peak for measurement, we are able to change the spectral fluctuations of the peak into intensity fluctuations due to the fluctuation of the emission in and out of the measurement window. Figure 3(a) shows the emission spectrum from our studied quantum dot along with a series of measurement windows (full peak, $1 / 4$ width, and $1 / 8$ width). The corresponding autocorrelation traces are presented in Fig. 3(b). These measurements were all performed under $375 \mathrm{~nm}$ excitation at a power of $72 \mathrm{~W} \mathrm{~cm}^{-2}$. The data are fitted with a typical model of an antibunched correlation with a single exponential bunching envelope. ${ }^{23}$

As we become more spectrally selective with our filter, a pronounced bunching can be seen in the autocorrelation, characterized by $g^{(2)}(\tau)>1$. The bunching appears because the data points for the autocorrelation histograms are acquired by the measurement of time differences between detected photons, and we are less likely to measure two photons with longer time differences because of an increasing chance that the spectral diffusion may have shifted the emission out of the measurement window at longer times. A measurement of the decay time in the bunching profile is thus a measurement of the characteristic time scale of the spectral diffusion ${ }^{41}$ or a measurement of how quickly the environment itself is fluctuating. In terms of the experiment, the bunching peak becomes increasingly visible for narrower spectral windows, with the requirement of longer data acquisition times (the time required to measure a given signal grows with the inverse square of the measured emission intensity). The widths of the selected spectral windows have no influence on the measured fast spectral diffusion time scales but only on the degree of bunching. ${ }^{41}$

It is important to note that the bunching appears only when we selectively measure a fraction of the peak and is therefore not due to the presence of a real 3-level system ${ }^{42}$ in this case. However, we note that the results are in fact compatible with such an analysis: For example, we could artificially assign 3 levels to the system [(1) QD is occupied and emission energy is within the filter window, (2) QD is occupied and emission energy is blocked by the spectral filter, and (3) QD is empty]. In this picture, the spectral diffusion process shifts the emitter between states 1 and 2 .

Analysis of the data in Fig. 3(b) reveals typical characteristic time scales of the bunching effect of $\sim 260 \mathrm{~ns}$, providing an upper limit of the time scale in within which indistinguishable photons could in principle be generated using these InGaN QDs (other sources of decoherence such as phonon interactions are likely to reduce this further). For time scales longer than this, environmental fluctuations will cause a change in the emission energy such that the emitted photons could in principle be distinguished by use of an optical system with a dispersive element. However, even though the energetic extent of the diffusion is large ( $\sim \mathrm{meV}$ levels-likely due to the large permanent dipole moment of the confined exciton ${ }^{22}$ ), the time scale of the fluctuations is slow enough that the successive emission of several photons should be possible between fluctuations in the environment (the emission lifetime of the dot is measured to be $\sim 1 \mathrm{~ns}$ ). We note that this spectral diffusion time scale of $260 \mathrm{~ns}$ is longer than values reported in the literature for some other typical semiconductor QDs, such as $\sim 10-20 \mathrm{~ns}$ in GaN/ $\mathrm{AlGaN},{ }^{23} \sim 5 \mathrm{~ns}$ in $\mathrm{CdSe} / \mathrm{ZnSe},{ }^{41,43}$ and comparable to some InGaAs/ GaAs QDs which exhibit time scales of 90-1900 ns. ${ }^{44}$ Although a direct comparison between different materials can be difficult, we note that the spectral diffusion times measured from these InGaN quantum dots are an order of magnitude longer than those measured in $\mathrm{GaN}$ QDs, ${ }^{23}$ despite the fact that the excitation power density is over an order of magnitude greater in this case. ${ }^{21,23}$ The relatively long spectral diffusion times measured here could be due to the existence of fairly deep charge traps (such that carrier escape and subsequent migration

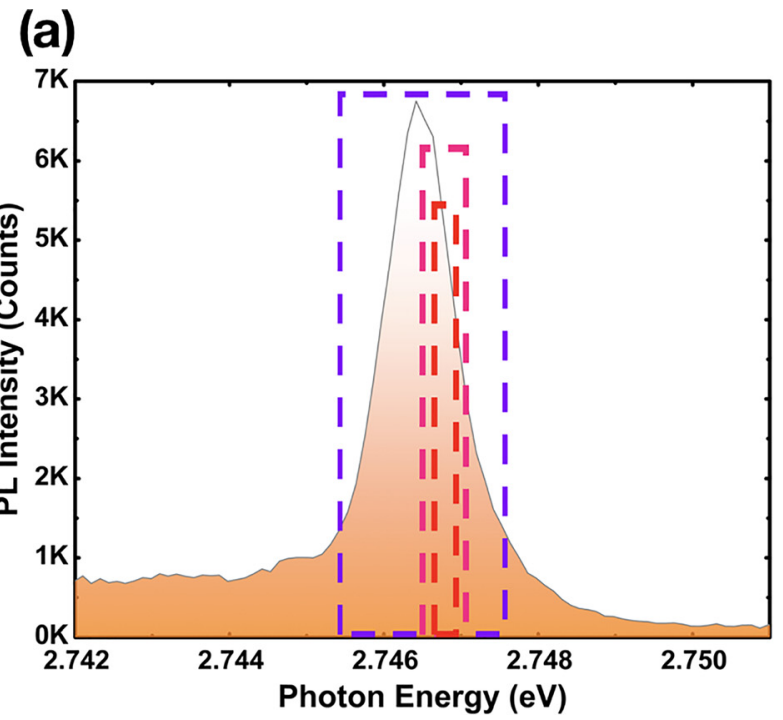

(b)

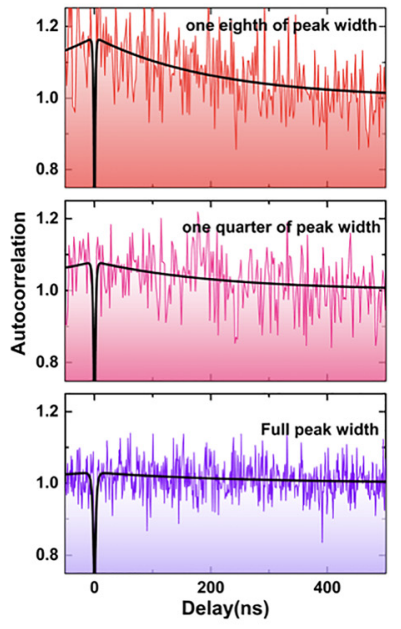

FIG. 3. Spectral diffusion measurements using autocorrelation under $375 \mathrm{~nm}$ excitation with a power of $72 \mathrm{~W} \mathrm{~cm}^{-2}$ (a) Photoluminescence spectrum with a series of chosen measurement regions. (b) Corresponding 2nd order autocorrelations of the measurement windows in (a). The apparent bunching is due to spectral diffusion in and out of the measurement windows. 
occur on long time scales). This may be somehow linked to the fragmentation of the quantum well that forms along with the QDs during the growth process or due to compositional fluctuations in the ternary material.

Next, we discuss the excitation wavelength and the power dependence of the time scale of the bunching. Similar to the long time scale diffusion shown in Fig. 2, we measure enhanced spectral diffusion for excitation into the barrier material. Indeed, as can be seen in Fig. 4(a), in which we present the inverse of the bunching time as a function of the excitation wavelength, we observe an increased spectral diffusion rate for excitation at $355 \mathrm{~nm}$ (with a corresponding bunching time of $\sim 100 \mathrm{~ns}$ ). As in the case of the long time scale measurement, the reason for this increase in the spectral diffusion rate here is due to an increased number of charge traps that can be excited by the $355 \mathrm{~nm}$ laser. With a larger number of excited electrons and possible trap states in the environment, it is more likely that the environment will change and cause a shift in the emission energy of the dot within a given time period. In Fig. 4(b), we present the power dependence of the spectral diffusion time scales under excitation of $375 \mathrm{~nm}$. The data reveal a more or less linear increase in the spectral diffusion rate with increasing power, in good agreement with studies on other materials, ${ }^{23,43,45}$ and supporting the notion that the spectral diffusion dynamics are governed by single carrier processes (rather than multiple carrier processes such as Auger-assisted carrier escape from traps).

In conclusion, we have performed experimental characterization of the excitation wavelength dependence of the spectral diffusion effects in a spectrally isolated single-photon emitting InGaN/GaN quantum dot. We observe spectral diffusion occurring on timescales of a few seconds and also on timescales of a few hundreds of nanoseconds. Although the underlying physics is the same (interactions with fluctuating charge distributions in the local environment), we believe that different time scales arise due to different distributions of traps with different characteristic trap times. We find that direct excitation into InGaN leads to both a suppression of the spectral extent of the spectral diffusion and also a slowing down of its time scale. The fastest spectral diffusion can occur on time scales larger than $250 \mathrm{~ns}$, which is one order of magnitude longer than that recently measured from $\mathrm{GaN}$ quantum dots (albeit with fluctuations that occur over a larger energy range). We have shown that even though the spectral diffusion limited linewidths of these emitters are broad, the time scales on which the
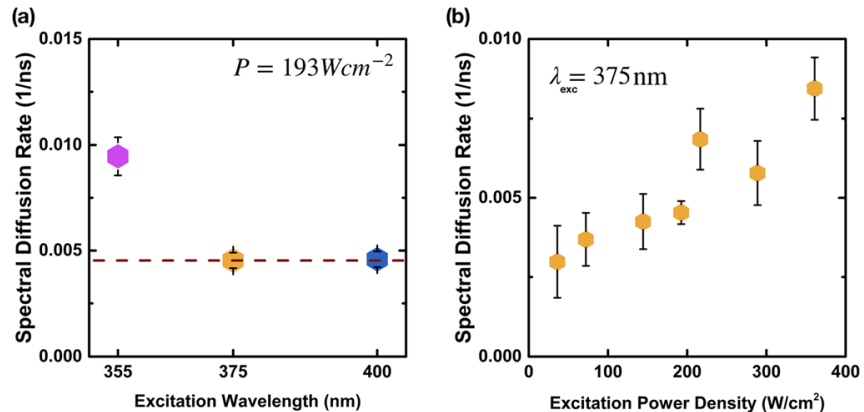

FIG. 4. (a) Spectral diffusion rates as a function of excitation wavelength (measurements performed at $7 \mathrm{~K}$ with an excitation power of $193 \mathrm{~W} \mathrm{~cm}^{-2}$ ). (b) Spectral diffusion rates as a function of excitation power (measurements performed at $7 \mathrm{~K}$ with an excitation wavelength of $375 \mathrm{~nm}$ ) diffusion occurs are slow enough that indistinguishable photon generation should, in principle, be possible, provided that other sources of decoherence could also be sufficiently suppressed. Indeed, the measurements described here reveal that the average time between the environmental fluctuations that give rise to the measured broadened linewidths can be as large as a few hundred nanoseconds, so that in principle (neglecting other sources of decoherence), a few hundred photons could be emitted at the same energy in the time windows between environmental fluctuations. It is likely that the spectral diffusion could be further suppressed by using resonant excitation of carriers directly into the emitting state.

This work was supported by a JSPS KAKENHI Grant-in-Aid for Specially Promoted Research (No. 15H05700), the KAKENHI project (No. 17K14655), the JSPS Summer Program, the Takuetsu program of the Ministry of Education, Culture, Sports, Science and Technology, Japan, and the UK Engineering and Physical Sciences Research Council Grant (No. EP/M011682/1). The authors thank X. Sun for technical support.

\section{REFERENCES}

${ }^{1}$ P. Michler, A. Kiraz, C. Becher, W. Schoenfeld, P. Petroff, L. Zhang, E. Hu, and A. Imamoglu, Science 290, 2282 (2000).

${ }^{2}$ J. C. Loredo, N. A. Zakaria, N. Somaschi, C. Anton, L. De Santis, V. Giesz, T. Grange, M. A. Broome, O. Gazzano, G. Coppola et al., Optica 3, 433 (2016).

${ }^{3}$ N. Somaschi, V. Giesz, L. De Santis, J. C. Loredo, M. P. Almeida, G. Hornecker, S. L. Portalupi, T. Grange, C. Anton, J. Demory et al., Nat. Photonics 10, 340 (2016).

${ }^{4}$ T. Miyazawa, K. Takemoto, Y. Nambu, S. Miki, T. Yamashita, H. Terai, M. Fujiwara, M. Sasaki, Y. Sakuma, M. Takatsu, T. Yamamoto, and Y. Arakawa, Appl. Phys. Lett. 109, 132106 (2016).

${ }^{5}$ X. Wang, X. Cai, Z. Su, M. Chen, D. Wu, L. Li, N. Liu, C. Lu, and J. Pan, Nature 518, 516 (2015)

${ }^{6}$ M. Kroutvar, Y. Ducommun, D. Heiss, M. Bichler, D. Schuh, G. Abstreiter, and J. Finley, Nature 432, 81 (2004).

${ }^{7}$ H. Kimble, Nature 453, 1023 (2008).

${ }^{8}$ E. Waks, K. Inoue, C. Santori, D. Fattal, J. Vuckovic, G. Solomon, and Y. Yamamoto, Nature 420, 762 (2002).

${ }^{9}$ B. Daudin, J. Phys: Condens. Matter 20, 473201 (2008).

${ }^{10}$ H. Sekiguchi, K. Kishino, and A. Kikuchi, Appl. Phys. Lett. 96, 231104 (2010).

${ }^{11}$ S. Tomic, J. Pal, M. Migliorato, R. Young, and N. Vukmirović, ACS Photonics 2, 958 (2015).

${ }^{12}$ B. Damilano, N. Grandjean, F. Semond, J. Massies, and M. Leroux, Appl. Phys. Lett. 75(7), 962 (1999).

${ }^{13}$ S. Kako, C. Santori, K. Hoshino, S. Götzinger, Y. Yamamoto, and Y. Arakawa, Nat. Mater. 5, 887 (2006)

${ }^{14}$ S. Deshpande, T. Frost, A. Hazari, and P. Bhattacharya, Appl. Phys. Lett. 105, 141109 (2014).

${ }^{15}$ M. Holmes, K. Choi, S. Kako, M. Arita, and Y. Arakawa, Nano Lett. 14, 982 (2014).

${ }^{16}$ S. Kako, M. Holmes, S. Sergent, M. Burger, D. As, and Y. Arakawa, Appl. Phys. Lett. 104, 011101 (2014).

${ }^{17}$ M. Holmes, S. Kako, K. Choi, M. Arita, and Y. Arakawa, ACS Photonics 3, 543 (2016).

${ }^{18}$ F. Le Roux, K. Gao, M. Holmes, S. Kako, M. Arita, and Y. Arakawa, Sci. Rep. 7, 16107 (2017)

${ }^{19}$ T. Wang, T. J. Puchtler, T. Zhu, J. C. Jarman, L. P. Nuttall, R. A. Oliver, and R. A. Taylor, Nanoscale 9, 9421 (2017).

${ }^{20}$ S. Gong, S. Kim, J. Kim, J. Cho, and Y. Cho, ACS Photonics 5, 711-717 (2018).

${ }^{21}$ M. Arita, F. Le Roux, M. Holmes, S. Kako, and Y. Arakawa, Nano Lett. 17, 2902 (2017).

${ }^{22}$ M. Holmes, S. Kako, K. Choi, M. Arita, and Y. Arakawa, Phys. Rev. B 92, 115447 (2015).

${ }^{23}$ K. Gao, I. Solovev, M. Holmes, M. Arita, and Y. Arakawa, AIP Adv. 7, 125216 (2017). 
${ }^{24}$ C. Kindel, G. Callsen, S. Kako, T. Kawano, H. Oishi, G. Honig, A. Schliwa, A. Hoffmann, and Y. Arakawa, Phys. Status Solidi RRL 8(5), 408 (2014).

${ }^{25}$ S. Sergent, S. Kako, M. Burger, T. Schupp, D. As, and Y. Arakawa, Appl. Phys. Lett. 105(14), 141112 (2014).

${ }^{26}$ R. Bardoux, T. Guillet, P. Lefebvre, T. Taliercio, T. Bretagnon, S. Rousset, B. Gil, and F. Semond, Phys. Rev. B 74, 195319 (2006).

${ }^{27}$ B. Reid, T. Zhu, T. Puchtler, L. Fletcher, C. Chan, R. Oliver, and R. Taylor, Jpn. J. Appl. Phys., Part 1 52, 08JE01 (2013).

${ }^{28}$ C. Santori, D. Fattal, J. Vuckovic, G. Solomon, and Y. Yamamoto, Nature 419, 594-597 (2002).

${ }^{29}$ H. Wang, Y. He, Y. Li, Z. Su, B. Li, H. Huang, X. Ding, M. Chen, C. Liu, J. Qin et al., Nat. Photonics 11, 361-365 (2017).

${ }^{30}$ T. Huber, A. Predojević, D. Föger, G. Solomon, and G. Weihs, New J. Phys. 17, 123025 (2015).

${ }^{31}$ T. Santana, Y. Ma, R. Malein, F. Bastiman, E. Clarke, and B. Gerardot, Phys. Rev. B 95, 201410(R) (2017).

${ }^{32}$ R. A. Oliver, G. A. Briggs, M. J. Kappers, C. J. Humphreys, S. Yasin, J. H. Rice, J. D. Smith, and R. A. Taylor, Appl. Phys. Lett. 83, 755 (2003).

${ }^{33}$ T. Zhu, F. Oehler, B. P. L. Reid, R. M. Emery, R. A. Taylor, M. J. Kappers, and R. A. Oliver, Appl. Phys. Lett. 102, 251905 (2013).

${ }^{34}$ T. Zhu, H. A. R. El-Ella, B. Reid, M. J. Holmes, R. A. Taylor, M. J. Kappers, and R. A. Oliver, J. Cryst. Growth 338, 262 (2012).
${ }^{35}$ H. P. Springbett, K. Gao, J. Jarman, T. Zhu, M. Holmes, Y. Arakawa, and R. A. Oliver, Appl. Phys. Lett. 113, 101107 (2018).

${ }^{36}$ T. Zhu, Y. Liu, T. Ding, W. Y. Fu, J. Jarman, C. X. Ren, R. Vasant Kumar, and R. A. Oliver, Sci. Rep. 7, 45344 (2017).

${ }^{37}$ A. Woolf, T. Puchtler, I. Aharonovich, T. Zhu, N. Niu, D. Wang, R. Oliver, and E. L. Hu, Proc. Natl. Acad. Sci. 111, 14042 (2014).

${ }^{38}$ J. H. Rice, J. W. Robinson, A. Jarjour, R. A. Taylor, R. A. Oliver, G. A. D. Briggs, M. J. Kappers, and C. J. Humphreys, Appl. Phys. Lett. 84, 4110 (2004).

${ }^{39}$ M. J. Holmes, M. Arita, and Y. Arakawa, Semicond. Sci. Technol. 34, 033001 (2019).

${ }^{40}$ S. A. Empedocles and M. G. Bawendi, Science 278, 2114 (1997).

${ }^{41} \mathrm{G}$. Sallen, A. Tribu, T. Aichele, R. André, L. Besombes, C. Bougerol, M. Richard, S. Tatarenko, K. Kheng, and J. Poizat, Nat. Photonics 4, 696 (2010).

${ }^{42}$ T. T. Tran, S. Choi, J. A. Scott, Z.-Q. Xu, C. Zheng, G. Seniutinas, A. Bendavid, M. S. Fuhrer, M. Toth, and I. Aharonovich, Adv. Opt. Mater. 5, 1600939 (2017).

${ }^{43} \mathrm{G}$. Sallen, A. Tribu, T. Aichele, R. André, L. Besombes, C. Bougerol, M. Richard, S. Tatarenko, K. Kheng, and J. Poizat, Phys. Rev. B 84, 041405(R) (2011).

${ }^{44}$ M. Abbarchi, T. Kuroda, T. Mano, M. Gurioli, and K. Sakoda, Phys. Rev. B 86, 115330 (2012).

${ }^{45}$ J. Wolters, N. Sadzak, A. Schell, T. Schroder, and O. Benson, Phys. Rev. Lett. 110, 027401 (2013). 